OPEN

SUBJECT AREAS:

THEORETICAL PHYSICS

QUANTUM OPTICS

QUANTUM INFORMATION

Received

17 February 2014

Accepted

9 April 2014

Published

28 April 2014

Correspondence and requests for materials should be addressed to H.F. (hfan@iphy.ac.cn) or W.-B.Y.

lyanweibin1983@ iphy.ac.cn)

\section{Single-photon quantum router with multiple output ports}

\author{
Wei-Bin Yan \& Heng Fan
}

Beijing National Laboratory for Condensed Matter Physics, Institute of Physics, Chinese Academy of Sciences, Beijing 100190, China.

The routing capability is a requisite in quantum network. Although the quantum routing of signals has been investigated in various systems both in theory and experiment, the general form of quantum routing with many output terminals still needs to be explored. Here we propose a scheme to achieve the multi-channel quantum routing of the single photons in a waveguide-emitter system. The channels are composed by the waveguides and are connected by intermediate two-level emitters. By adjusting the intermediate emitters, the output channels of the input single photons can be controlled. This is demonstrated in the cases of one output channel, two output channels and the generic $\mathrm{N}$ output channels. The results show that the multi-channel quantum routing of single photons can be well achieved in the proposed system. This offers a scheme for the experimental realization of general quantum routing of single photons.

Q uantum routing ${ }^{1-9}$ of information from one sender to many receivers is an essential function for the quantum network ${ }^{10}$. Single photons are suitable candidates as the carrier of quantum information due to the fact that they propagate fast and interact rarely with the environment. The field-matter interaction can be used to manipulate the photons. By the way, the light-light interaction by the platform of linear optics ${ }^{8,9,11,12}$ can also be used to manipulate the photons. The photon transport in a one dimensional (1D) waveguide has been studied extensively both in theory ${ }^{13-26}$ and in experiments ${ }^{27-36}$ because the strong coupling of the waveguideemitter can be achieved. In the waveguide-emitter system, the waveguides act as the channels and the emitters as the nods of the quantum network. Based on these advantages, the quantum routing of photons in the waveguideemitter system is promising. Recently, two output channel quantum routing of single photons is studied theoretically in a waveguide-emitter system ${ }^{6,7}$. The input single photons can be redirected into either of the two output channels with a maximal probability of unity and no more than $1 / 2$, respectively. It is interesting if the photon can be redirected into either of the output channels with an extremely high probability. Moreover, a general form of many output channel quantum routing of the single photons in the waveguide QED system will be of considerable interest.

For these purposes, we propose a novel scheme for the quantum routing of single photons from one input waveguide into $\mathrm{N}$ output waveguides. In our scheme, the ith output waveguide is connected with the input waveguide by an intermediate two-level system (TLS). After scattering, the single photon injected into the input channel is redirected into other channels. For the single output channel quantum routing, the quantum interferences redirect the input photon into the output channel completely when the intermediate TLS resonantly interacts with the input and output channels with the same strength. The single output channel routing properties can be modified when an additional TLS is coupled to the input channel. In the two output channel case, the photon can be redirected into either of the output channels with an approximate unity probability. The photon can also be redirected completely into both the output channels with various probabilities. In the generic $\mathrm{N}$ output channel case, the quantum interferences prevent the photon being redirected into the other channels except the input channel when all the TLSs resonantly interact with the channels with equal strengths for a large value of $\mathrm{N}$. They also can completely prevent the photon being directed back into the input channel for suitable parameters for any value of $\mathrm{N}$. The photon can be redirected into a desired channel with an approximate unity probability.

The schematic diagram of the system under consideration is shown in Fig. 1. The input channel is a Sagnac interferomete ${ }^{37-39}$ composed by a semi-infinite waveguide, a 50:50 beam splitter and a waveguide loop. The input channel connects the $i$ th infinite waveguide by an intermediate TLS at the middle point of the waveguide loop. For simplicity, we label the interaction position $x=0$. It is convenient to bring in the even and odd operators as $a_{e k}=\frac{1}{\sqrt{2}}\left(a_{r k}+a_{l k}\right)$ and $a_{o k}=\frac{1}{\sqrt{2}}\left(a_{r k}-a_{l k}\right)$, with $a_{r k}\left(a_{l k}\right)$ being the annihilation operator for the right (left)- 


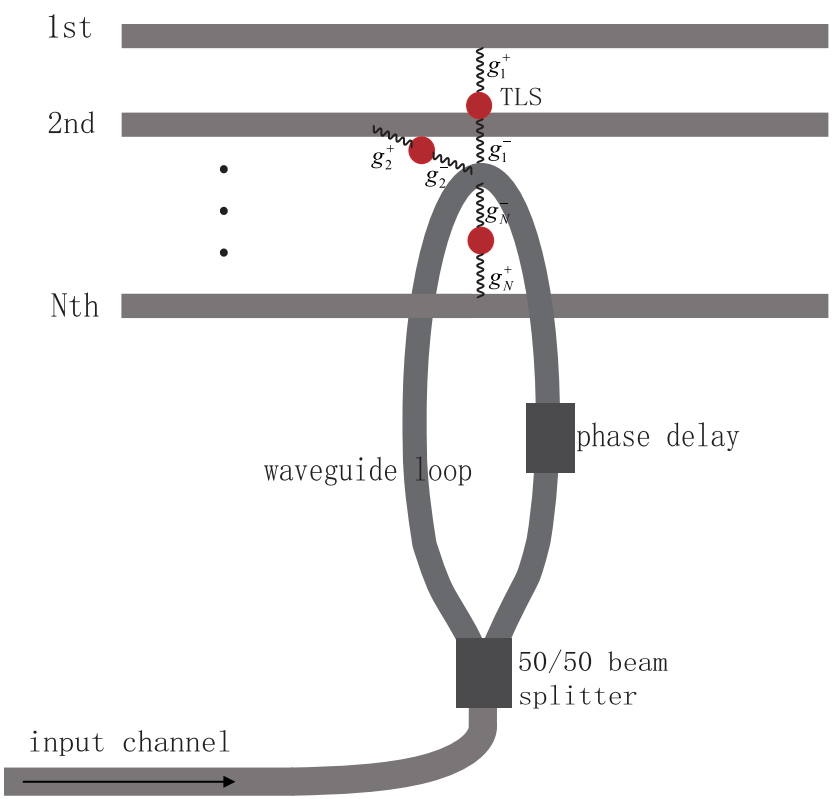

Figure 1 Schematic diagram of the multi-channel quantum routing of the single photons. N1D waveguides are connected with the input channel by $N$ intermediate TLSs.

moving mode with the frequency $v_{g}|k|$ in the waveguide. Hereafter we will take the photon group velocity $v_{g}=1$. The clockwise- and counterclockwise-moving photons in the waveguide loop can be treated as the left- and right-moving photons. If we inject a photon into the input waveguide, an even mode quasi particle can be produced at the middle point of the waveguide loop when the phases of the clockwise- and counter clockwise-moving photons are equal. In the even and odd picture, the odd mode only contributes to the free energy part in the Hamiltonian. Therefore, it is enough to study the dynamics of the even mode. For simplicity, we omit the superscript $e$ of the even operator $a_{e k}$. The even part of the Hamiltonian can be written as $(\hbar=1)$

$$
\begin{aligned}
H= & \int_{-\infty}^{\infty} d k k a_{k}^{\dagger} a_{k}+\sum_{i}\left[\int_{-\infty}^{\infty} d k k a_{i, k}^{\dagger} a_{i, k}+\omega_{i} \sigma_{i}^{+} \sigma_{i}^{-}\right. \\
& \left.+\int_{-\infty}^{\infty} d k\left(g_{i}^{+} a_{i, k}+g_{i}^{-} a_{k}\right) \sigma_{i}^{+}+\text {h.c. }\right],
\end{aligned}
$$

with $a_{k}^{\dagger}\left(a_{i, k}^{\dagger}\right)$ being the even mode creation operator in the input (ith output) waveguide, and $\omega_{i}$ being the $i$ th TLS transition frequency. We have taken the energies of the TLS ground states zero. The terms of the second line in Hamiltonian (1) represent the interaction of the TLSs with the waveguides. $\frac{g_{i}^{-}}{\sqrt{2}}$ and $\frac{g_{i}^{+}}{\sqrt{2}}$ are coupling strengths of the $i$ th TLS to the input waveguide and the $i$ th output waveguide, respectively. The coupling strengths can be written as $g_{i}^{\zeta}=\sqrt{\frac{\gamma_{i}^{\zeta}}{2 \pi}}(\zeta= \pm)$, with $\gamma_{i}^{\zeta}$ being the decay rate from the $i$ th TLS to the waveguides. The TLS can be a real two-level atom or an artificial two-level system. In the single-photon case, the TLS can also be a cavity or a cavity-atom dressed system. The transition frequency of the TLS can be tuned by Stark shift and the coupling strength between the waveguide and the TLS can be varied by changing the distance between them ${ }^{40,41}$. Especially, for a waveguide-atom dressed system, the effective coupling strength of the dressed TLS to the waveguide can be adjusted by the extra laser ${ }^{42}$. We have made two approximations: one is to extend the frequency integration to $\pm \infty$, the other is to assume that the coupling strengths are independent of the frequencies, which is equivalent to the Markovian approximation. These approximations are valid since we will focus on the pulse with a narrow frequency width around the carrier frequency.

The arbitrary state of the system in the single-excitation subspace has the form of $\left|\Psi^{(N)}(t)\right\rangle=\int d k \alpha_{k}^{(N)}(t) a_{k}^{\dagger}+\sum_{i}\left[\int d k \alpha_{i, k}^{(N)}(t) a_{i, k}^{\dagger}+\right.$ $\left.\beta_{i}^{(N)}(t) \sigma_{i}^{+}\right]|\phi\rangle$, with $\alpha_{k}^{(N)}(t), \alpha_{i, k}^{(N)}(t)$ and $\beta_{i}^{(N)}(t)$ being the probability amplitudes. The state $|\phi\rangle$ represents that all the waveguides and TLSs contain no excitation. We assume that, initially, a photon prepared in a wave packet with a Lorenzian spectrum is injected into the input waveguide, while the TLSs and the output waveguides contain no excitation, i.e. $\alpha_{k}^{(N)}(0)=\frac{\sqrt{\epsilon / \pi}}{k-\varpi+i \epsilon}, \alpha_{i, k}^{(N)}(0)=0, \beta_{i}^{(N)}(0)=0$. Here $\epsilon$ and are the spectral width and the center frequency of the input wave packet, respectively. When the width $\epsilon \rightarrow 0$, the frequency of the carrier is equal to the central frequency. This corresponds to the monochromatic limit.

\section{Results}

Single output channel. When the input channel is connected with only one output channel by a TLS, the probability amplitudes are $\alpha_{1, k}^{(1)}=\frac{-\sqrt{\gamma_{1}^{-} \gamma_{1}^{+}}}{i \delta_{1}+\frac{\gamma_{1}^{+}}{2}+\frac{\gamma_{1}^{-}}{2}}$, and $\alpha_{k}^{(1)}=\frac{i \delta_{1}+\frac{\gamma_{1}^{+}}{2}-\frac{\gamma_{1}^{-}}{2}}{i \delta_{1}+\frac{\gamma_{1}^{+}}{2}+\frac{\gamma_{1}^{-}}{2}}$, with $\delta_{i}=\omega_{i}-k$. Obviously, only when $\frac{\gamma_{1}^{+}}{\gamma_{1}^{-}}=1$ and $\delta_{1}=0$, the input photon is redirected into the output channel completely. This is due to the quantum interferences. When the detuning $\delta_{1}$ is large enough, the photon will be back into the input channel with an approximate unity probability. Here we bring in a parameter $\eta_{i}=\frac{\max \left\{\gamma_{i}^{+}, \gamma_{i}^{-}\right\}}{\min \left\{\gamma_{i}^{+}, \gamma_{i}^{-}\right\}}$to measure the difference between the coupling strengths of the $i$ th TLS to the input and the $i$ th waveguides. When $\eta_{1} \gg 1$, we can get $\left|\alpha_{k}^{(1)}\right|^{2} \approx 1$, which is similar to the large detuning case. Although the decay rate of the TLS to the input channel is much smaller than the decay rate to the output channel, the photon is prevented being redirected into the output channel. The condition $\eta_{1} \rightarrow \infty$ corresponds to the limit that one of the two waveguides is decoupled to the TLS. In this case, the input and output channels are not connected, and the photon will be back into the input channel completely. To show the details of the quantum routing of the single phonon in the $N=1$ case, we plot the probability $\left|\alpha_{1, k}^{(1)}\right|^{2}$ against the detuning and decay rates in Fig. 2. The probability $\left|\alpha_{k}^{(1)}\right|^{2}$ is not plotted here because $\left|\alpha_{k}^{(1)}\right|^{2}=1-\left|\alpha_{1, k}^{(1)}\right|^{2}$. The probabilities of the

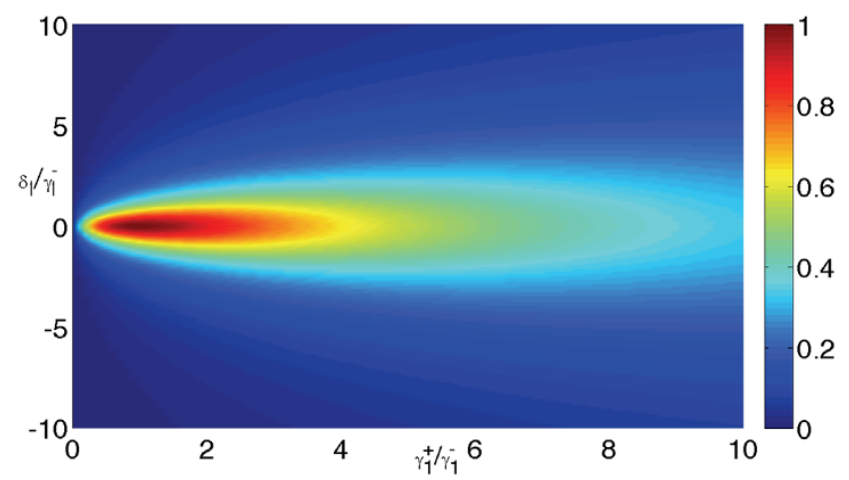

Figure $2 \mid$ Probability of the single photon in the 1st output channel in the long-time limit $\left|\alpha_{1, k}^{(1)}\right|^{2}$ against the detuning and decay rate when $N=1$. 
photon in the input and output channels can be controlled by adjusting the detuning and coupling strengths. The single photon can be directed into either of the two channels completely or directed into the two channels with a desired probability. The $N=1$ case is analogous to a waveguide coupled to a $\Lambda$-type three-level quantum emitter ${ }^{25,43,44}$.

Two output channels. When the input channel is connected with two output channels by two TLSs, the probability amplitudes are obtained as $\alpha_{1, k}^{(2)}=\frac{-\sqrt{\gamma_{1}^{-} \gamma_{1}^{+}}\left(i \delta_{2}+\frac{\gamma_{2}^{+}}{2}\right)}{\left(i \delta_{1}+\frac{\gamma_{1}^{+}}{2}+\frac{\gamma_{1}^{-}}{2}\right)\left(i \delta_{2}+\frac{\gamma_{2}^{+}}{2}+\frac{\gamma_{2}^{-}}{2}\right)-\frac{\gamma_{1}^{-} \gamma_{2}^{-}}{4}}$, and $\alpha_{k}^{(2)}=\frac{\left(i \delta_{1}+\frac{\gamma_{1}^{+}}{2}-\frac{\gamma_{1}^{-}}{2}\right)\left(i \delta_{2}+\frac{\gamma_{2}^{+}}{2}-\frac{\gamma_{2}^{-}}{2}\right)-\frac{\gamma_{1}^{-} \gamma_{2}^{-}}{4}}{\left(i \delta_{1}+\frac{\gamma_{1}^{+}}{2}+\frac{\gamma_{1}^{-}}{2}\right)\left(i \delta_{2}+\frac{\gamma_{2}^{+}}{2}+\frac{\gamma_{2}^{-}}{2}\right)-\frac{\gamma_{1}^{-} \gamma_{2}^{-}}{4}} . \quad$ Because the expression of $\alpha_{1, k}^{(2)}$ is similar to $\alpha_{2, k}^{(2)}$, the study of the properties $\left|\alpha_{1, k}^{(2)}\right|^{2}$ and $\left|\alpha_{k}^{(2)}\right|^{2}$ is enough. The large detuning between the 1st TLS and the input photon prevents the photon being redirected into the 1st channel. Especially, when $\delta_{2}=0$ and $\gamma_{2}^{+}=\gamma_{2}^{-}$, it also prevents the photon being directed into the input channel. As a result, for an extremely large value of $\delta_{1}$, the photon will be redirected into the 2 nd channel with an approximate unity probability when $\delta_{2}=0$ and $\gamma_{2}^{+}=\gamma_{2}^{-}$. And when both the detunings $\delta_{1}$ and $\delta_{2}$ are large enough, the photon will be directed into the input channel with an approximate unity probability. Besides, the photon distribution can be influenced significantly by the coupling strengths. For example, when $\delta_{2}=0$ and $\gamma_{2}^{+}=\gamma_{2}^{-}$, the large value of $\gamma_{1}^{+}$prevents the photon being directed into both the input and 1st channels. When $\eta_{1}=\eta_{2}=1$ and $\delta_{1}=\delta_{2}=0$, the input photon will be directed into the input channel with a small probability and be redirected into the other two channels averagely with a large probability. To see the details, we plot the probabilities $\left|\alpha_{1, k}^{(2)}\right|^{2}$ and $\left|\alpha_{k}^{(2)}\right|^{2}$ against the detuning and coupling strength of the 2nd TLS when $\delta_{1}=0$ and $\gamma_{1}^{+}=\gamma_{1}^{-}=\gamma_{2}^{-}$in Fig. 3(a) and 3(b). It is interesting that when $\delta_{2}$ and $\gamma_{2}^{+}$is small enough, the photon will be back into the input channel with an extremely high probability. This will be studied in detail below.
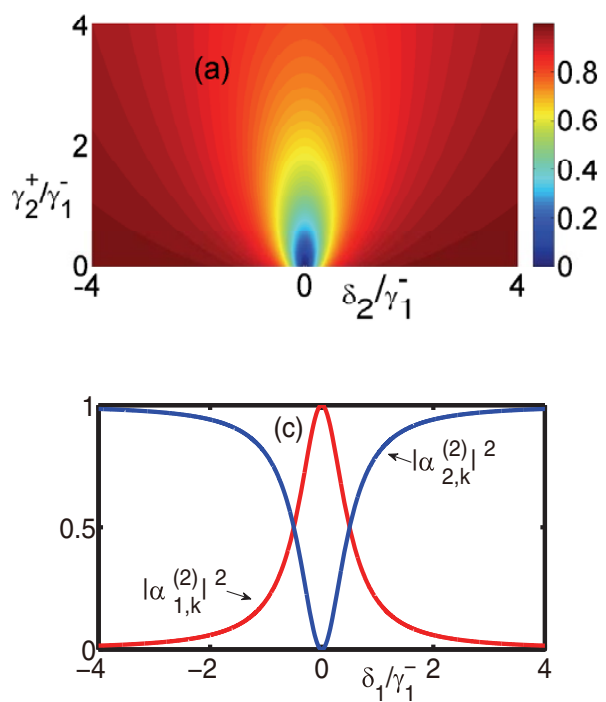

If we do not wish the input photon to be back into the input channel, it is easy to choose the appropriate parameters which satisfy $\alpha_{k}^{(2)}=0$. Here we take two simple cases to investigate it. One case is when $\gamma_{1}^{+}=\gamma_{1}^{-}$and $\gamma_{2}^{+}=\gamma_{2}^{-}$, the condition $4 \delta_{1} \delta_{2}+\gamma_{1}^{-} \gamma_{2}^{-}=0$ can be satisfied for various values of the detunings. The other case is when $\delta_{1}$ $=\delta_{2}=0$, the relation $\left(\gamma_{1}^{+}-\gamma_{1}^{-}\right)\left(\gamma_{2}^{+}-\gamma_{2}^{-}\right)=\gamma_{1}^{-} \gamma_{2}^{-}$can be easily satisfied. Especially, in the latter case, when $\gamma_{1}^{+}=2 \gamma_{1}^{-}$and $\gamma_{2}^{+}=2 \gamma_{2}^{-}$, the input single photon is redirected into the two output channels with equal probability $\frac{1}{2}$. We plot the probabilities of the photon being redirected into each of the two output channels in the two cases in Fig. 3(c) and 3(d). It shows that the input photon can be completely redirected into the output channels with various probabilities.

It is necessary to study a special case of $N=2$, that is, the 2nd TLS is decoupled to the 2 nd output channel, i.e. $\gamma_{2}^{+}=0$. This can be understood that an additional TLS is coupled to the input channel in the $N=1$ case. The additional TLS will modify the system behavior. For example, when $\eta_{1}=1$ and $\delta_{1}=0$, we find $\alpha_{1, k}^{(2)}=\frac{-i 4 \delta_{2} \gamma_{1}^{-}}{4 i \delta_{2} \gamma_{1}^{-}+\gamma_{1}^{-} \gamma_{2}^{-}}$and $\alpha_{k}^{(2)}=\frac{-\gamma_{1}^{-} \gamma_{2}^{-}}{4 i \delta_{2} \gamma_{1}^{-}+\gamma_{1}^{-} \gamma_{2}^{-}}$. Hence, when the additional TLS resonantly interacts with the input photon, i.e. $\delta_{2}=0$, the photon is directed into the input channel compared with the $N=1$ case, in which the photon is redirected into the 1 st output channel. This can be seen in Fig. 3(a) and 3(b). When $\delta_{2}$ is large enough, the input photon will be almost completely redirected, mapped to the $N=1$ case. By adjusting the additional TLS, the photon distribution in the input and output channels can be controlled. This provides more control to the single output channel case.

$N$ output channels. We proceed to study the general case that the input channel connects with $N$ output channels by NTLSs. Let's first consider the simplest case that all of the $N$ output channels are identical. That is to say, all the TLS are identical, all the decay rates to the input channel are identical, and all the decay rates to the output channels are identical. We label $\delta_{i}=\delta, \gamma_{i}^{+}=\gamma^{+}$, and $\gamma_{i}^{-}=\gamma^{-}$. The probability amplitudes are obtained as $\alpha_{i, k}^{(N)}=\frac{-2 \sqrt{\gamma^{-} \gamma^{+}}}{2 i \delta+\gamma^{+}+N \gamma^{-}}$, and $\alpha_{k}^{(N)}=\frac{2 i \delta+\gamma^{+}-N \gamma^{-}}{2 i \delta+\gamma^{+}+N \gamma^{-}}$. It is noted that when all the TLSs interact
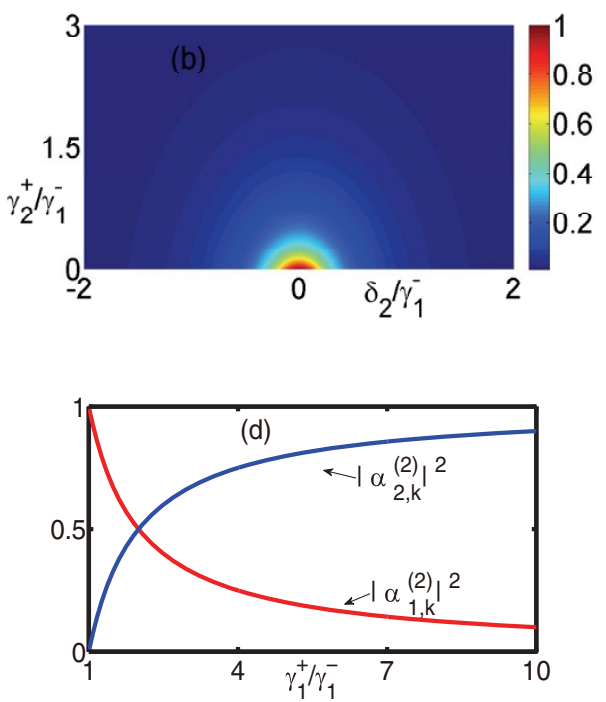

Figure $3 \mid$ Probabilities $\left|\alpha_{1, k}^{(2)}\right|^{2},\left|\alpha_{2, k}^{(2)}\right|^{2}$ and $\left|\alpha_{k}^{(2)}\right|^{2}$ against the detunings and coupling strengthes when $N=2$. (a) and (b) are the probabilities $\left|\alpha_{1, k}^{(2)}\right|^{2}$ and $\left|\alpha_{k}^{(2)}\right|^{2}$ when $\delta_{1}=0$ and $\gamma_{1}^{-}=\gamma_{1}^{+}=\gamma_{2}^{-}$, respectively. (c) and (d) are the probabilities $\left|\alpha_{1, k}^{(2)}\right|^{2}$ and $\left|\alpha_{2, k}^{(2)}\right|^{2}$. (c) is the probabilities against the detuning $\delta_{1}$ when $\gamma_{1}^{+}=\gamma_{1}^{-}, \gamma_{2}^{+}=\gamma_{2}^{-}$, and $4 \delta_{1} \delta_{2}+\gamma_{1}^{-} \gamma_{2}^{-}=0$. (d) is the probabilities against the coupling constant $\gamma_{1}^{+}$when $\delta_{1}=\delta_{2}=0$, and $\left(\gamma_{1}^{+}-\gamma_{1}^{-}\right)\left(\gamma_{2}^{+}-\gamma_{2}^{-}\right)=\gamma_{1}^{-} \gamma_{2}^{-}$. 
resonantly with the input photon and the coupling strengths satisfy $\gamma^{+}=N \gamma^{-}$, the interferences prevent the input photon being directed into the input channel and redirect the input photon into each of the $N$ output channels with equal probability $\frac{1}{N}$. When all the coupling strengths are equal and all the detunings are zero, the probabilities are obtained as $\left|\alpha_{i, k}^{(N)}\right|^{2}=\left(\frac{2}{1+N}\right)^{2}$ and $\left|\alpha_{k}^{(N)}\right|^{2}=\left(\frac{1-N}{1+N}\right)^{2}$. As discussed above, the quantum interferences redirect the input photon completely from the input channel into the other channel when $N=1$. As the number of the output channels increases, the probability of the photon back into the input channel increases. When $N=3$, the input photon is distributed in each of the four channels, including the input and output channels, with equal probability $\frac{1}{4}$. When the number of the output channels is large enough, the quantum interferences direct the input photon back into the input channel almost completely.

We now study a simple case to illustrate how to redirect the input photon into the desired channel. We assume that $N-1$ of the $N$ channels are identical except the $m$ th channel. we label $\delta_{i \neq m}=\delta^{\prime}$, $\gamma_{i \neq m}^{+}=\gamma^{+\prime}$, and $\gamma_{i \neq m}^{-}=\gamma^{-\prime}$. The probability amplitudes are obtained as $\quad \alpha_{m, k}^{(N)}=\frac{-\sqrt{\gamma_{m}^{-} \gamma_{m}^{+}}\left(i \delta^{\prime}+\frac{\gamma^{+\prime}}{2}\right)}{\left(i \delta_{m}+\frac{\gamma_{m}^{+}}{2}\right)\left(i \delta^{\prime}+\frac{\gamma^{+\prime}}{2}+\frac{(N-1) \gamma^{-\prime}}{2}\right)+\frac{\gamma_{m}^{-}}{2}\left(i \delta^{\prime}+\frac{\gamma^{+\prime}}{2}\right)}$ and $\alpha_{k}^{(N)}=\frac{\left(i \delta_{m}+\frac{\gamma_{m}^{+}}{2}\right)\left(i \delta^{\prime}+\frac{\gamma^{+\prime}}{2}-\frac{(N-1) \gamma^{-\prime}}{2}\right)-\frac{\gamma_{m}^{-}}{2}\left(i \delta^{\prime}+\frac{\gamma^{+\prime}}{2}\right)}{\left(i \delta_{m}+\frac{\gamma_{m}^{+}}{2}\right)\left(i \delta^{\prime}+\frac{\gamma^{+\prime}}{2}+\frac{(N-1) \gamma^{-\prime}}{2}\right)+\frac{\gamma_{m}^{-}}{2}\left(i \delta^{\prime}+\frac{\gamma^{+\prime}}{2}\right)}$.

When the decay rates $\gamma_{m}^{-}=\gamma_{m}^{+}$are much larger than other parameters, $\alpha_{m, k}^{(N)} \approx-\frac{i \delta^{\prime}+\frac{\gamma^{+\prime}}{2}}{i \delta^{\prime}+\frac{\gamma^{+\prime}}{2}+\frac{(N-1) \gamma^{-\prime}}{4}}$. The photon will be redirected into the $m$ th channel almost completely when $2 \gamma^{+\prime} \gg(N-1) \gamma^{-\prime}$. The limit of this condition is that the $N-1$ TLSs are decoupled to the input channel. Besides, when all the detunings are large enough, the photon will be directed into the input channel with an approximate unity probability. When $\gamma_{m}^{-}=\gamma_{m}^{+}, \delta_{m}=0$, and $\delta^{\prime}$ is large enough, the photon will be redirected into the $m$ th channel with an approximate unity probability. Similar to the $N=2$ case, the photon can be redirected completely into the output channels where the photon probabilities can be controlled. As a consequence, the $N$ output channel quantum routing of the single photons can be achieved.

The single photons are considered as the ideal carrier of quantum information. The quantum information can be encoded by the routed photons. We label two degenerate photon modes as $a_{1}$ and $a_{2}$. For example, the two degenerate modes can be horizontally and vertically polarized modes or can be right and left circularly polarized modes. To achieve the quantum routing of the quantum information, we bring in $N$ extra intermediate TLSs to make sure that each output port is connected with the input port by two intermediate TLSs. The distance between the two intermediate TLSs is much smaller than the wavelength of the photon. One of the two intermediate TLSs only interacts with $a_{1}$-mode photon and the other only interacts with $a_{2}$ mode photon. In this case, the Hamiltonian can be written as $H=\sum_{m=1,2}\left\{\int_{-\infty}^{\infty} d k k a_{m k}^{\dagger} a_{m k}+\sum_{i}\left[\int_{-\infty}^{\infty} d k k a_{m i, k}^{\dagger} a_{m i, k}+\omega_{m i} \sigma_{m i}^{+}\right.\right.$ $\left.\left.\sigma_{m i}^{-}+\int_{-\infty}^{\infty} d k\left(g_{m i}^{+} a_{m i, k}+g_{m i}^{-} a_{m k}\right) \sigma_{m i}^{+}+h . c.\right]\right\}$. To study the quantum routing of the quantum information, we take the input state as $|\Psi\rangle=\left[A \alpha_{k}^{(N)}(0) a_{1 k}^{\dagger}+B \alpha_{k}^{(N)}(0) a_{2 k}^{\dagger}\right]|\phi\rangle$, with $|A|^{2}+|B|^{2}=1$. Obviously, when $g_{1 i}^{+}=g_{2 i}^{+}, g_{1 i}^{-}=g_{2 i}^{-}$and $\omega_{1 i}=\omega_{2 i}$, the input state $|\Psi\rangle$ can be routed to the desired channel with desired probability. In this case, the information remains unchanged after being routed. In our scheme, the control information is stored in the coupling strength and the TLSs. It will be interesting to use quantum information to control the routing. This might be achieved by preparing connections between the control quantum states and the target quantum states.

The authors in Ref. 9 have raised a set of five requirements imposed on a full quantum router. It will be interesting when a router meets all the requirements. As discussed above, Our scheme will meet all those requirements if the control information can also be encoded into quantum states. This full quantum routing scheme in various systems is an interesting question which need be further studied.

\section{Discussion}

We have investigated the quantum routing of the single photons from one channel to multiple output channels in the proposed system. We have shown that the input photon can be redirected into any of the channels with a high efficiency in our scheme. Alternatively, by cascaded combining many two output channel routers demonstrated in the presented literatures, the multiple output channel quantum routing can be achieved. We note that our general $N$ output channel quantum routing shows essential advantages compared with the cascaded combined one. In the cascaded combined scheme, the efficiency of the $i$ output channel quantum routing is limited by the efficiency of the $i-1$ output channel quantum routing. However, our scheme is immune to these drawbacks. It is necessary to point out that our scheme is equivalent to that the input waveguide loop is coupled to $N$ output waveguide by an intermediate $N+1$-level system at the middle point of the waveguide loop. Our scheme sheds light on the experimental realization of quantum routing.

\section{Methods}

The continuum form of Hamiltonian (1) can be derived from its discrete form by following the technique in Ref. 45, 46. The free Hamiltonian of the photon in the 1D cavity of length $L$ has the form of $H_{0}=\sum_{k} \omega_{k} \tilde{a}_{k}^{\dagger} \tilde{a}_{k}$, and the interaction Hamiltonian of the photon with the TLS has the form of $H_{I}=\sum_{k} \tilde{g} \tilde{a}_{k}^{\dagger} \sigma^{-}+h . c$. The sum can be converted into an integral by $\frac{2 \pi}{L} \sum_{k} \rightarrow \int d k$. As done in Ref. 45 , we can bring in the operator $a_{k}^{\dagger}=\sqrt{\frac{L}{2 \pi}} \tilde{a}_{k}^{\dagger}$ and obtain the Hamiltonian (1) with continuum form.

The Shrödinger equation governed by Hamiltonian (1) gives a set of differential equations of probability amplitudes. By performing the Laplace transformation, the set of differential equations under the initial condition are transformed as

$$
\begin{aligned}
\alpha_{k}^{(N)}(s) & =\frac{\sqrt{\pi / \epsilon}}{\left(s+i \omega_{k}\right)\left(\omega_{k}-\varpi+i \epsilon\right)}-\sum_{j=1 . . N} \frac{i g_{j}^{-}}{s+i \omega_{k}} \beta_{j}^{(N)}(s) \\
\alpha_{i, k}^{(N)}(s) & =\frac{-i g_{i}^{+}}{s+i \omega_{i, k}} \beta_{i}^{(N)}(s) \\
0 & =\left(s+i \omega_{i}+\frac{\gamma_{i}^{+}}{2}\right) \beta_{i}^{(N)}(s)+\sum_{j=1 . . N} \frac{\sqrt{\gamma_{i}^{-} \gamma_{j}^{-}}}{2} \beta_{j}^{(N)}(s)+\frac{2 \pi g_{i}^{-} \sqrt{\pi / \epsilon}}{s+\epsilon+i \varpi}
\end{aligned}
$$

We proceed to obtain the expression of $\beta_{i}^{(N)}(s)$. For simplicity, we label $a_{i}=s+i \omega_{i}+\frac{\gamma_{i}^{+}}{2}, b_{i}=\sqrt{\frac{\gamma_{i}^{-}}{2}}$, and $c_{i}=\frac{2 \pi g_{i}^{-} \sqrt{\pi / \epsilon}}{s+\epsilon+i \varpi}$. The last equation in equations (2) can be written as

$$
a_{i} \beta_{i}^{(N)}(s)+b_{i} \sum_{j=1 . . N} b_{j} \beta_{j}^{(N)}(s)+c_{i}=0
$$

From equation (3), we can find $\sum_{j=1 . . N} b_{j} \beta_{j}^{(N)}(s)=-\sum_{i=1 . . N} \frac{b_{i}^{2}}{a_{i}}$ $\sum_{j=1 . . N} b_{j} \beta_{j}^{(N)}(s)-\sum_{i=1 \ldots N} \frac{c_{i} b_{i}}{a_{i}}$ to obtain the expression of $\sum_{j=1 . . N} b_{j} \beta_{j}^{(N)}$. Consequently, the expression of $\beta_{i}^{(N)}(s)$ can be obtained as

$$
\beta_{i}^{(N)}=-\frac{2 \pi \sqrt{\pi / \epsilon}}{s+\epsilon+i \varpi} \frac{g_{i}^{-} \prod_{j \neq i}\left(s+i \omega_{j}+\gamma_{j}^{+}\right)}{\prod_{j=1 . . N}\left(s+i \omega_{j}+\gamma_{j}^{+}\right)+\sum_{j=1 . . N}\left[\gamma_{j}^{-} \prod_{j^{\prime} \neq j}\left(s+i \omega_{j^{\prime}}+\gamma_{j^{\prime}}^{+}\right)\right]}(4)
$$

From the equation (4) and equations (2), the expressions of $\alpha_{k}^{(N)}(s)$ and $\alpha_{i, k}^{(N)}(s)$ can be easily found. 
In the long-time limit, the amplitudes can be found by performing the inverse Laplace transformation of $\alpha_{k}^{(N)}(s), \alpha_{i, k}^{(N)}(s)$ and $\beta_{i}^{(N)}(s)$. Then we prove that the amplitude $\beta_{i}^{(N)}(t) \approx 0$ when $t \rightarrow \infty$. It means that the TLSs are in their ground states in the long-time limit due to the interaction with the continuum of modes. Obviously, $\prod_{j=1 . . N}\left(s+i \omega_{j}+\gamma_{j}^{+}\right)+\sum_{j=1 . . N}\left[\gamma_{j}^{-} \prod_{j^{\prime} \neq j}\left(s+i \omega_{j^{\prime}}+\gamma_{j^{\prime}}^{+}\right)\right]=\prod_{i=1 . . N}\left(s-a_{i}\right)$, with $a_{i}$ being the root of the equation

$\prod_{j=1 . . N}\left(s+i \omega_{j}+\gamma_{j}^{+}\right)+\sum_{j=1 . . N}\left[\gamma_{j}^{-} \prod_{j^{\prime} \neq j}\left(s+i \omega_{j^{\prime}}+\gamma_{j^{\prime}}^{+}\right)\right]=0$. We lable $a_{i}=x_{i}$ $+i y_{i}$, with $x_{i}$ and $y_{i}$ being real numbers. We will show that the value of $x_{i}$ can only be smaller than zero.

If $x_{i} \geq 0$, we can get

$\prod_{j=1 . . N}\left(a_{i}+i \omega_{j}+\gamma_{j}^{+}\right)+\sum_{j=1 . . N}\left[\gamma_{j}^{-} \prod_{j^{\prime} \neq j}\left(a_{i}+i \omega_{j^{\prime}}+\gamma_{j^{\prime}}^{+}\right)\right]$

$=\prod_{j=1 . . N}\left(i m_{j}+n_{j}\right)+\sum_{j=0 . . N}\left[\gamma_{j}^{-} \prod_{j^{\prime} \neq j}\left(i m_{j^{\prime}}+n_{j^{\prime}}\right)\right]=0$, with $m_{j}$ and $n_{j}$ being

real numbers and $n_{j}>0$. We label $i m_{j}+n_{j}=R_{j} e^{i \theta_{j}}$ and $\Pi_{j=1 . N}\left(i m_{j}+n_{j}\right)=R e^{i \theta}$, and get

$$
\prod_{j=1 . . N}\left(i m_{j}+n_{j}\right)+\sum_{j=1 . . N}\left[\gamma_{j}^{-} \prod_{j^{\prime} \neq j}\left(i m_{j^{\prime}}+n_{j^{\prime}}\right)\right]=R e^{\theta}+\sum_{j=1 . . N} \gamma_{j}^{-} \frac{R}{R_{j}} e^{i\left(\theta-\theta_{j}\right)} .
$$

Obviously, it can not be zero because $\frac{\pi}{2}>\theta_{j} \geq 0$ or $2 \pi \geq \theta_{j}>\frac{3 \pi}{2}$, and

$\left\{\gamma_{j}^{-}, R, R_{j}\right\}>0$. Therefore, the value of $x_{i}$ must be smaller than 0 . As a consequence, we can find $\beta_{i}^{(N)}(t)=0$.

Similarly, the probability amplitudes of the photon in each channel in the longtime limit are found as $\alpha_{i, k}^{(N)}(t)=\alpha_{i, k}^{(N)} \alpha_{k}^{(N)}(0) e^{-i k t}$ and $\alpha_{k}^{(N)}(t)=\alpha_{k}^{(N)} \alpha_{k}^{(N)}(0) e^{-i k t}$,
$\lim t \rightarrow \infty$ with

$$
\begin{gathered}
\alpha_{i, k}^{(N)}=\frac{-\sqrt{\gamma_{i}^{-} \gamma_{i}^{+}} \prod_{j \neq i}\left(i \delta_{j}+\frac{\gamma_{j}^{+}}{2}\right)}{\prod_{j=1 . . N}\left(i \delta_{j}+\frac{\gamma_{j}^{+}}{2}\right)+\sum_{j=1 . . N}\left[\frac{\gamma_{j}^{-}}{2} \prod_{j^{\prime} \neq j}\left(i \delta_{j^{\prime}}+\frac{\gamma_{j}^{+}}{2}\right)\right]}, \\
\alpha_{k}^{(N)}=\frac{\prod_{j=1 . . N}\left(i \delta_{j}+\frac{\gamma_{j}^{+}}{2}\right)-\sum_{j=1 . . N}\left[\frac{\gamma_{j}^{-}}{2} \prod_{j^{\prime} \neq j}\left(i \delta_{j^{\prime}}+\frac{\gamma_{j}^{+}}{2}\right)\right]}{\prod_{j=1 . . N}\left(i \delta_{j}+\frac{\gamma_{j}^{+}}{2}\right)+\sum_{j=1 . . N}\left[\frac{\gamma_{j}^{-}}{2} \prod_{j^{\prime} \neq j}\left(i \delta_{j^{\prime}}+\frac{\gamma_{j}^{+}}{2}\right)\right]} .
\end{gathered}
$$

We have taken the conversation of energy condition $\omega_{i, k}=\omega_{k}$. After scattering, the probabilities of the single photon in each channel are $\left|\alpha_{i, k}^{(N)}\right|^{2}$ and $\left|\alpha_{k}^{(N)}\right|^{2}$.

1. Aoki, T. et al. Efficient routing of single photons by one atom and a microtoroidal cavity. Phys. Rev. Lett. 102, 083601 (2009).

2. Hoi, I.-C. et al. Demonstration of a single-photon router in the microwave regime. Phys. Rev. Lett. 107, 073601 (2011).

3. Agarwal, G. S. \& Huang, S. Optomechanical systems as single-photon routers. Phys. Rev. A 85, 021801(R) (2012).

4. Ma, X., Zotter, S., Kofler, J., Jennewein, T. \& Zeilinger, A. Experimental generation of single photons via active multiplexing. Phys. Rev. A 83, 043814 (2011).

5. Xia, K. \& Twamley, J. All-optical switching and router via the direct quantum control of coupling between cavity modes. Phys. Rev. X 3, 031013 (2013).

6. Zhou, L., Yang, L. P., Li, Y. \& Sun, C. P. Quantum routing of single photons with a cyclic three-level system. Phys. Rev. Lett. 111, 103604 (2013).

7. Lu, J., Zhou, L., Kuang, L. M. \& Nori, F. Single-photon router: Coherent control of multichannel scattering for single photons with quantum interferences. Phys. Rev. A 89, 013805 (2013).

8. Lemr, K. \& Černoch, A. Linear-optical programmable quantum router. Opt Comm. 300, 282 (2013).

9. Lemr, K., Bartkiewicz, K., Černoch, A. \& Soubusta, J. Resource-efficient linearoptical quantum router. Phys. Rev. A 87, 062333 (2013).

10. Kimble, H. J. The quantum internet. Nature 453, 1023 (2008).

11. Passaro, E. et al. Joining and splitting the quantum states of photons preprint at http://arxiv.org/abs/1309.4254 (2013).

12. Vitelli, C. et al. Joining the quantum state of two photons into one. Nature Photon. 7, 521 (2013).

13. Shen, J.-T. \& Fan, S. Coherent photon transport from spontaneous emission in one-dimensional waveguides. Opt. Lett. 30, 2001 (2005).

14. Shen, J.-T. \& Fan, S. Strongly correlated two-photon transport in a onedimensional waveguide coupled to a two-Level system. Phys. Rev. Lett. 98, 153003 (2007).

15. Shen, J.-T. \& Fan, S. Strongly correlated multiparticle transport in one dimension through a quantum impurity. Phys. Rev. A, 76, 062709 (2007).

16. Zhou, L., Gong, Z. R., Liu, Y.-X., Sun, C. P. \& Nori, F. Controllable scattering of a single photon inside a one-dimensional resonator waveguide. Phys. Rev. Lett. 101, 100501 (2008).
17. Roy, D. Two-photon scattering by a driven three-level emitter in a onedimensional waveguide and electromagnetically induced transparency. Phys. Rev. Lett. 106, 053601(2011)

18. Rephaeli, E. \& Fan, S. Stimulated emission from a single excited atom in a waveguide. Phys. Rev. Lett. 108, 143602(2012).

19. Longo, P., Schmitteckert, P \& Busch, K. Few-photon transport in low-dimensional systems: interaction-induced radiation trapping. Phys. Rev. Lett. 104, 023602 (2010).

20. Zheng, H., Gauthier, D. J. \& Baranger, H. U. Cavity-free photon blockade induced by many-body bound states. Phys. Rev. Lett. 107, 223601(2011).

21. Zheng, H. \& Baranger, H. U. Persistent quantum beats and long-distance entanglement from waveguide-mediated interactions. Phys. Rev. Lett. 110, 113601 (2013).

22. Zheng, H. \& Baranger, H. U. Waveguide-QED-based photonic quantum computation. Phys. Rev. Lett. 111, 090502 (2013).

23. Liao, J. Q. \& Law, C. K. Correlated two-photon transport in a one-dimensional waveguide side-coupled to a nonlinear cavity. Phys. Rev. A 82, 053836 (2010).

24. Huang, J. F., Shi, T., Sun, C. P. \& Nori, F. Controlling single-photon transport in waveguides with finite cross section. Phys. Rev. A 88, 013836 (2013).

25. Witthaut, D. \& Sørensen, A. S. Photon scattering by a three-level emitter in a onedimensional waveguide. New J. Phys. 12, 043052 (2010).

26. Li, Q., Zhou, L. \& Sun, C. P. Waveguide QED: controllable channel from quantum interference. preprint at http://arxiv.org/abs/1308.2011 (2013).

27. Dayan, B. et al. A photon turnstile dynamically regulated by one atom. Science 319, 1062 (2008).

28. Vetsh, E. et al. Optical interface created by laser-cooled atoms trapped in the evanescent field surrounding an optical nanofiber. Phys. Rev. Lett. 104, 203603 (2010).

29. Akimov, A. V. et al. Generation of single optical plasmons in metallic nanowires coupled to quantum dots. Nature (London) 450, 402 (2007).

30. Bajcsy, M. et al. Efficient all-optical switching using slow light within a hollow fiber. Phys. Rev. Lett. 102, 203902 (2009).

31. Babinec, T. M. et al. A diamond nanowire single-photon source. Nature Nanotechnol. 5, 195 (2010)

32. Claudon, J. et al. A highly efficient single-photon source based on a quantum dot in a photonic nanowire. Nature Photon. 4, 174 (2010).

33. Astafiev, O. et al. Resonance fluorescence of a single artificial atom. Science 327, 840 (2010).

34. Astafiev, O. et al. Ultimate on-chip quantum amplifier. Phys. Rev. Lett. 104, 183603 (2010).

35. Bleuse, J. et al. Inhibition, enhancement, and control of spontaneous emission in photonic nanowires. Phys. Rev. Lett. 106, 103601 (2011).

36. Laucht, A. et al. A waveguide-coupled on-chip single-photon source. Phys. Rev. X 2, 011014 (2012).

37. Menon, V. M. et al. All-optical wavelength conversion using a regrowth-free monolithically integrated Sagnac interferometer. IEEE Photonics Technol. Lett. 15, 254 (2003).

38. Menon, V. M., Tong, W., Xia, F., Li, C. \& Forrest, S. R. Nonreciprocity of counterpropagating signals in a monolithically integrated Sagnac interferometer. Opt. Lett. 29, 513 (2004).

39. Bertocchi, G., Alibart, O., Ostrowsky, D. B., Tanzilli, S. \& Baldi, P. Single-photon sagnac interferometer. J. Phys. B 39, 1011 (2006).

40. Chang, D. E., Sørensen, A. S., Hemmer, P. R. \& Lukin, M. D. Strong coupling of single emitters to surface plasmons. Phys. Rev. B 76, 035420 (2006).

41. Armani, D. K., Kippenberg, T. J., Spillane, S. M. \& Vahala, K. J. Ultra-high-Q toroid microcavity on a chip. Nature 421, 925 (2002).

42. Yan, W.-B., Fan, Q.-B. \& Zhou, L. Control of correlated two-photon transport in a one-dimensional waveguide. Phys. Rev. A 85, 015803 (2012).

43. Bradford, M., Obi, K. C. \& Shen, J.-T. Efficient single-photon frequency conversion using a sagnac interferometer. Phys. Rev. Lett. 108, 103902 (2012).

44. Bradford, M. \& Shen, J.-T. Single-photon frequency conversion by exploiting quantum interference. Phys. Rev. A 85, 043814 (2012).

45. Fan, S., Kocabaş, Ş. E. \& Shen, J.-T. Input-output formalism for few-photon transport in one-dimensional nanophotonic waveguides coupled to a qubit. Phys. Rev. A 82, 063821 (2010).

46. Blow, K. J., Loudon, R., Phoenix, S. J. D. \&Shepherd, T. J. Continuum fields in quantum optics. Phys. Rev. A 42, 4102 (1990).

\section{Acknowledgments}

This work is supported by "973" program (2010CB922904), NSFC (11175248), and grants from Chinese Academy of Sciences.

\section{Author contributions}

W.-B.Y. and H.F. proposed the model, calculates and analyzed the results. W.-B.Y. and H.F. co-wrote the paper.

\section{Additional information}

Competing financial interests: The authors declare no competing financial interests. 
How to cite this article: Yan, W.-B. \& Fan, H. Single-photon quantum router with multiple output ports. Sci. Rep. 4, 4820; DOI:10.1038/srep04820 (2014).

(c) (i) This work is licensed under a Creative Commons Attribution 3.0 Unported License The images in this article are included in the article's Creative Commons license, unless indicated otherwise in the image credit; if the image is not included under the Creative Commons license, users will need to obtain permission from the license holder in order to reproduce the image. To view a copy of this license, visit http://creativecommons.org/licenses/by/3.0/ 